\title{
Efeitos de duas técnicas de incentivo respiratório na mobilidade toracoabdominal após cirurgia abdominal alta
} Effects of two respiratory incentive techniques on chest wall mobility after upper abdominal surgery

\author{
Maria Elaine Trevisan', Juliana Corrêa Soares², Tatiana Zacarias Rondinel ${ }^{2}$
}

Estudo desenvolvido no Curso de Fisioterapia, Depto. de Fisioterapia e Reabilitação da UFSM - Universidade Federal de Santa Maria, Santa Maria, RS, Brasil

1 Profa. Ms. assistente do Depto. de Fisioterapia e Reabilitação da UFSM

2 Fisioterapeuta

ENDEREÇO PARA CORRESPONDÊNCIA:

M. Elaine Trevisan

R. Benjamin Constant 670 ap301 97050-022 Santa Maria RS e-mail: elaine.trevisan@yahoo. com.br

APRESENTAÇÃO

dez. 2009

ACEITO PARA PUBLICAÇÃO ago. 2010
Resumo: A cirurgia abdominal alta está associada a um risco elevado de complicações pulmonares que podem ser reduzidas pelo uso criterioso de manobras terapêuticas visando a expansão pulmonar. $\mathrm{O}$ objetivo foi comparar duas técnicas de incentivo respiratório na recuperação da dinâmica toracoabdominal em pacientes submetidos à cirurgia abdominal alta. O grupo de estudo experimental foi constituído por 16 pacientes internados na Clínica Cirúrgica do Hospital Universitário de Santa Maria distribuídos aleatoriamente em dois grupos: o grupo 1 foi constituído por dez pacientes que usaram o dispositivo Voldyne e o grupo 2, por seis pacientes submetidos ao padrão ventilatório com inspiração fracionada em três tempos. A expansibilidade toracoabdominal foi avaliada por cirtometria antes da cirurgia e no $1 \underline{0}, 3 \underline{0}$,

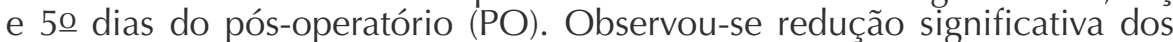
valores de cirtometria no $1 \underline{\text { o }} \mathrm{PO}$ que, gradualmente, foram sendo recuperados, não mais havendo diferença significativa no $5 \underline{0}$ PO em relação aos valores pré-operatórios em ambos os grupos. O grupo 1 obteve significativamente melhores índices de recuperação da mobilidade toracoabdominal do que o grupo 2. Também o tempo de recuperação do grupo 1 atingiu médias mais elevadas durante todo o período de PO investigado. Embora ambas as técnicas utilizadas fossem efetivas, o incentivo inspiratório por meio do Voldyne mostrou melhores resultados na recuperação da expansibilidade pulmonar após cirurgia abdominal alta.

Descritores: Cuidados pós-operatórios; Exercícios respiratórios; Modalidades de fisioterapia

Abstract: Upper abdominal surgery is associated to increased risk of pulmonary complications, which may be lessened by judicious use of therapeutic maneuvers aimed at lung expansion. The purpose here was to compare two respiratory incentive techniques on recovery of thoracic-abdominal dynamics in patients having undergone upper abdominal surgery. Sixteen patients in such condition were randomly divided into group $1(n=10)$, who did respiratory training with the Voldyne device, and group 2 $(n=6)$, submitted to a split-inspiration pattern training. Chest wall expansion was rated by measuring thorax circumferences before surgery and on the $1 \mathrm{st}$, 3rd, and 5th postoperative (PO) days. In both groups a significant decrease was found in circumference values on the 1 st $\mathrm{PO}$ day, which gradually recovered, until on the 5 th $\mathrm{PO}$ day no significant differences were found as compared to pre-operative measures. Group 1 showed significantly better thoracic-abdominal expansion rates than group 2's, as well as higher recovery time rates all through. Though both breathing techniques used were effective, inspiratory incentive using the Voldyne device showed better results in recovering chest mobility after upper abdominal surgery.

Key words: Breathing exercises; Postoperative care; Physical therapy modalities 
INTRODUÇÃO

As cirurgias do compartimento abdominal superior estão associadas a um risco elevado de complicações pulmonares, cuja gravidade pode ser reduzida pelo uso criterioso de manobras terapêuticas que visam a expansão pulmonar ${ }^{1-3}$. Esse tipo de incisão leva à ruptura de fibras dos músculos respiratórios que, associada ao quadro doloroso, podem ser responsáveis pela diminuição da atividade respiratória, gerando hipoventilação 4 . O procedimento cirúrgico do abdome superior leva também à redução dos volumes e capacidades pulmonares, modificação do modelo ventilatório, alteração da relação ventilação/perfusão, diminuição da função diafragmática, diminuição da expansibilidade toracoabdominal, ineficácia nos mecanismos de defesa, como a tosse, e depressão do sistema imunológico 5,6 .

A fisioterapia respiratória por meio de técnicas de higiene brônquica, expansão pulmonar, deambulação precoce e fortalecimento muscular tem sido usada com o propósito de prevenir o acúmulo de secreções e promover a melhora da ventilação, reduzindo a incidência de infecções pulmonares pós-operatórias, devendo ser iniciada desde o pré-operatório ${ }^{7-11}$. Apesar de o grande número de recursos terapêuticos descritos na literatura demonstrarem a eficácia na recuperação da função pulmonar e na redução das complicações pós-operatórias, nenhum método isolado é aceito como superior, ou que dispense a presença do fisioterapeuta 12,13 .

Sabe-se, no entanto, que técnicas ou instrumentos que encorajem o paciente a inspirar profundamente, como os padrões ventilatórios e a espirometria de incentivo, têm importância clínica e são largamente utilizados 12-14. Postula-se que esforços respiratórios máximos levariam à reinsuflação de regiões colapsadas do pulmão, pelo aumento do gradiente de pressão transpulmonar, podendo prevenir ou reverter atelectasias $6,14,15$.

Um dos tipos de incentivo respiratório freqüentemente utilizado com esse propósito é o Voldyne, aparelho orientado a volume que proporciona um estímulo visual ao paciente. Tem indicação no pós-operatório (PO) de cirurgia abdomi- nal, torácica e cardíaca, tendo em vista que sua utilização possibilita a realização de exercícios respiratórios com menor trabalho e menor estimulação dolorosa15. Outra possibilidade é o padrão ventilatório em três tempos, que consiste em inspiração fracionada: inspiração interrompida por pausa entre os três volumes inspirados e, ao final, expiração ao nível do repouso.

A dinâmica toracoabdominal é observada por cirtometria, que consiste em um conjunto de medições das circunferências de tórax e abdome durante os movimentos respiratórios, para avaliar a expansibilidade torácica de forma simples e acessível16.

O objetivo deste estudo foi comparar as técnicas de padrão ventilatório em três tempos e a espirometria de incentivo inspiratório na recuperação da dinâmica toracoabdominal em pacientes submetidos à cirurgia abdominal alta.

\section{METODOLOGIA}

O protocolo de pesquisa foi aprovado pelo Comitê de Ética em Pesquisa com Seres Humanos da Universidade Federal de Santa Maria e todos os voluntários formalizaram a participação no estudo assinando o termo de consentimento livre e esclarecido.

Os grupos do estudo experimental foram compostos por pacientes de ambos os sexos, na faixa dos 35 aos 75 anos, submetidos à cirurgia abdominal alta eletiva aberta, internados na Clínica Cirúrgica do Hospital Universitário de Santa Maria. Os critérios de exclusão foram cirurgia laparoscópica, presença de doença neurológica e/ou pulmonar prévia, tabagismo, deficit cognitivo, nível de consciência inadequado para realização de atividades orientadas, necessidade de sedação e extubação no período superior a 24 horas após a cirurgia.

Por ordem de internação, os pacientes foram distribuídos aleatoriamente em dois grupos, 1 e 2, iniciando pelo grupo 1 . Os pacientes de ambos os grupos foram avaliados no pré-operatório utilizando-se uma ficha de avaliação incluindo dados de identificação, história da doença pregressa e atual, hábitos sociais, histórico cirúrgico, indicadores de gravidade (tempo de cirurgia e índice ASA17) e medidas de cirtometria. Para a cirtometria utilizou-se uma fita métrica de material não-distensível. Foram mensurados os perímetros torácicos nos níveis axilar, xifóide e basal e o perímetro abdominal no nível da cicatriz umbilical16,18. Utilizou-se o incentivo verbal para que o paciente realizasse inspiração e/ou expiração máxima, direcionando o ar para a região avaliada 18 . Solicitou-se ao paciente uma expiração máxima seguida de uma inspiração profunda e lenta, momento em que se verificou e foi anotado o valor da circunferência inspiratória e, na seqüência, solicitou-se uma expiração máxima, momento em que foi verificado e anotado o valor da circunferência expiratória, em cada nível de medição. O coeficiente respiratório foi calculado pela diferença entre os dois valores mensurados. Esse procedimento foi repetido três vezes para cada região do tórax e abdome, sendo considerado o maior valor obtido. A cirtometria foi realizada no pré-operatório

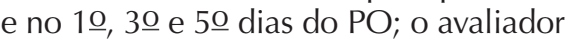
não tinha conhecimento do grupo a que pertencia o paciente.

Diariamente, durante todo o período de internação, os pacientes receberam terapia de higiene brônquica e, depois, terapia específica para cada grupo, uma vez ao dia. Durante a utilização do dispositivo Voldyne (grupo 1), incentivador respiratório a volume (Sherwood Medical, St Louis, $\mathrm{MO}$, USA), o paciente foi recostado a $45^{\circ}$ sendo solicitada uma inspiração lenta e profunda pelo bocal do aparelho. Foram efetuadas três séries de cinco inspirações profundas em cada série, com intervalo para descanso.

Para a execução do padrão ventilatório com inspiração fracionada em três tempos (grupo 2), o paciente foi recostado a $45^{\circ}$ e instado a realizar três séries de cinco respirações em cada série, com intervalo para descanso.

No 1무 $3 \underline{\mathrm{o}}$ e $5 \underline{\mathrm{o}}$ dias do pós-operatório, após a aplicação do Voldyne (grupo 1) ou do padrão ventilatório em três tempos (grupo 2), os pacientes tiveram um período de descanso de aproximadamente 10 minutos e, na seqüência, foram tomadas as medidas de circunferência como descrito acima.

Os dados foram submetidos à estatística descritiva e sua normalidade foi 
verificada pelo teste Shapiro-Wilk. Foi utilizado o teste não-paramétrico Mann-Whitney para a comparação entre os grupos e o teste para variáveis pareadas de Wilcoxon para comparação intra-grupo entre dois períodos. O pacote estatístico computacional utilizado foi o SAS (versão 8.2) e, para todos os testes, o nível de significância adotado foi de 5\% $(p=0,05)$.

\section{RESULTADOS}

A caracterização da amostra é apresentada na Tabela 1. Nenhum paciente requereu cuidados de terapia intensiva.

Tabela 1 Caracterização da amostra

\begin{tabular}{lcc}
\hline Característica & $\begin{array}{c}\text { Grupo 1 } \\
(\mathrm{n}=10)\end{array}$ & $\begin{array}{c}\text { Grupo 2 } \\
(\mathrm{n}=6)\end{array}$ \\
\hline Sexo F/M & $3 / 7$ & $4 / 2$ \\
Idade (anos)* & $37,4 \pm 11,57$ & $61,2 \pm 5,03$ \\
Tipo de cirurgia & & \\
$\quad$ Gastrectomia total & 3 & - \\
$\quad$ Hemicolectomia & 3 & 2 \\
$\quad$ Colecistectomia & 4 & 3 \\
$\quad$ Antrectomia & - & 1 \\
Tipo de incisão & & \\
$\quad$ Mediana supraumbilical & 6 & 6 \\
$\quad$ Subcostal & 4 & - \\
Tempo cirúrgico (horas) & $3,0 \pm 0,62$ & $3,2 \pm 0,41$ \\
Índice ASA ASA II & 6 & 3 \\
$\quad$ ASA III & 4 & 3 \\
\hline
\end{tabular}

$\mathrm{F}=$ feminino; $\mathrm{M}=$ masculino; * média \pm desvio padrão; ASA = classificação do estado físico da Sociedade Americana de Anestesiologia

Comparando as médias da expansibilidade nos níveis estudados, observou-se melhora na expansibilidade torácica e abdominal após a intervenção, tanto no grupo submetido a incentivo respiratório com o Voldyne quanto com o padrão ventilatório em três tempos. $\mathrm{Na}$ comparação entre os grupos, observou-se melhora significativa no grupo 1 nos níveis xifóide no 50 $\mathrm{PO}(p=0,01-$ Gráfico $1)$, costal no $3 \underline{0} \mathrm{PO}(p=0,01)$ e $5 \underline{\text { o }} \mathrm{PO}$ $(p=0,01$ - Gráfico 2) e umbilical no $5 \underline{0}$ PO ( $p=0,02$ - Gráfico 3).

Todos os pacientes apresentaram circunferências toracoabdominais menores no pós-operatório imediato, comparadas às dos pré-operatório. Comparando-se os valores médios do pré-operatório e $1 \underline{0}$ PO nos dois grupos, a diferença foi estatisticamente significativa no grupo 1 nos níveis axilar $(p=0,007)$, xifóide $(p=0,01)$, costal $(p=0,001)$ e umbilical $(p=0,01)$; e,

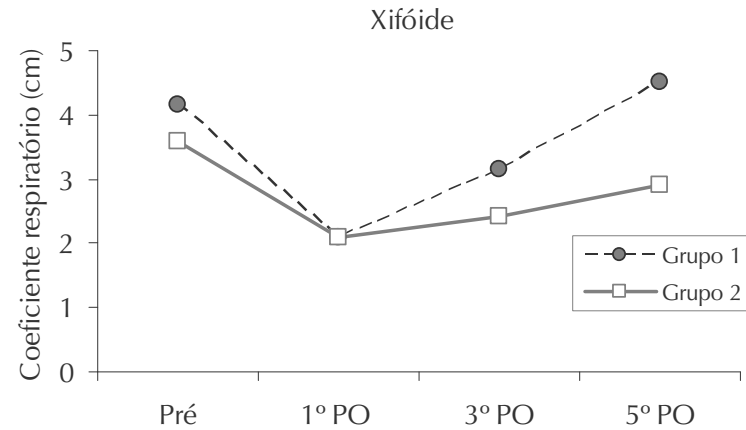

Gráfico 1 Coeficiente respiratório médio no nível xifóide dos grupos $1(n=10)$ e $2(n=6)$, medido no pré-operatório (Pré) e no 1으, 3o e 5으 dias de pós-operatório (PO)

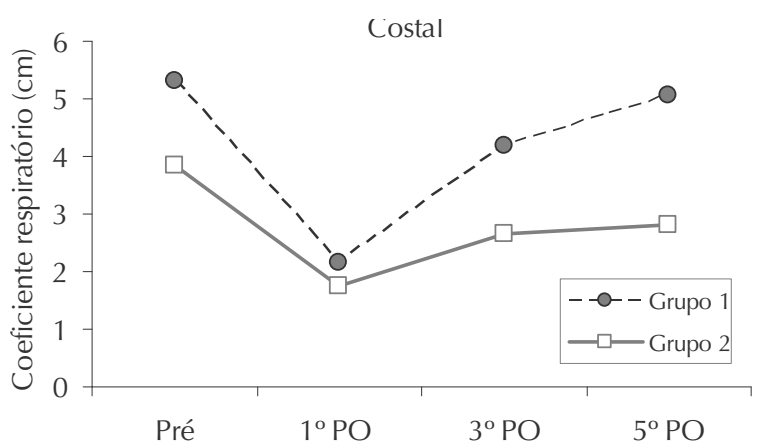

Gráfico 2 Coeficiente respiratório médio no nível costal dos grupos $1(n=10)$ e $2(n=6)$, medido no pré-operatório (Pré) e no 1으, 3으 e 50 dias de pós-operatório (PO)

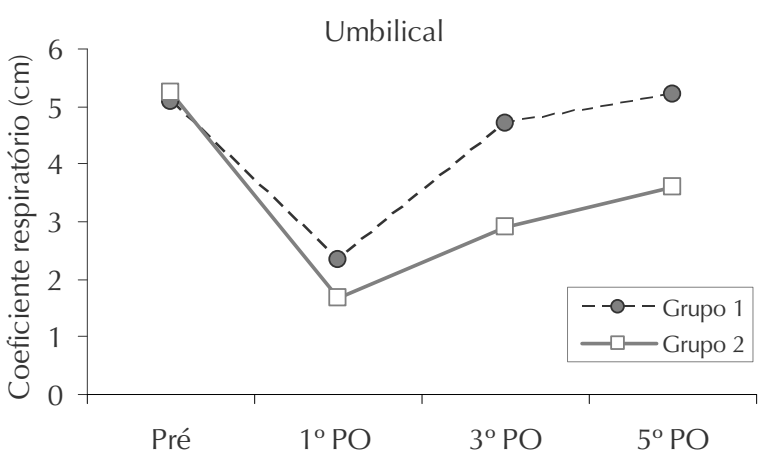

Gráfico 3 Coeficiente respiratório médio no nível umbilical dos grupos $1(n=10)$ e $2(n=6)$, medido no pré-operatório (Pré) e no 1으, 3으 e 5으 dias de pós-operatório (PO

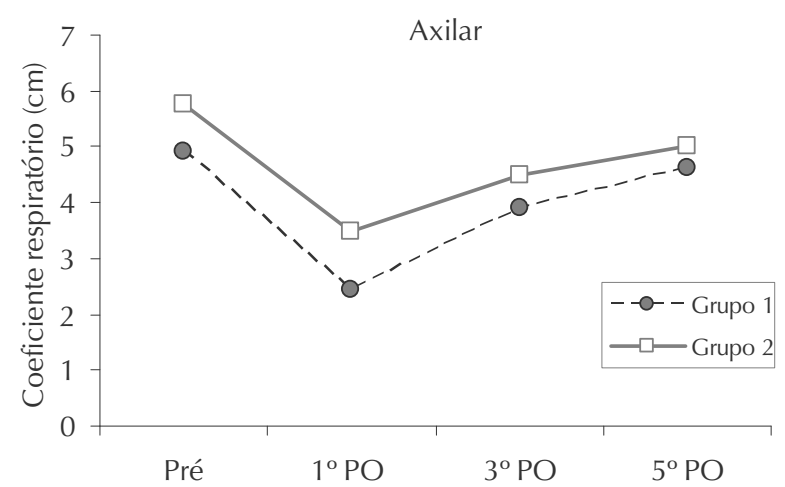

Gráfico 4 Coeficiente respiratório médio no nível axilar dos grupos $1(n=10)$ e $2(n=6)$, medido no pré-operatório (Pré) e no

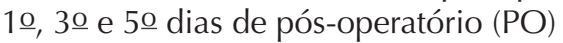


no grupo 2 , nos níveis axilar $(p=0,03)$, xifóide $(p=0,02)$ e umbilical $(p=0,01)$. Os percentuais de perda observadas no grupo 1 foram 50\% no nível axilar, 49,4\% no xifóide, 59,4\% no costal e 53,9\% no umbilical; no grupo 2 as perdas foram de $39,1 \%$ no axilar, $41,9 \%$ no xifóide, $54,3 \%$ no costal e $68,4 \%$ no umbilical.

Quando comparados o 1ㅇ e o 5으 dias PO, observa-se que as circunferências foram significativamente maiores nos níveis axilar $(p=0,003)$, xifóide $(p=0,005)$, costal $(p=0,003)$ e umbilical $(p=0,005)$ no grupo 1 ; e, no grupo 2, apenas nos níveis costal $(p=0,03)$ e umbilical $(p=0,02)$ no Grupo 2 .

$\mathrm{Na}$ comparação entre os grupos, observou-se que não diferiram significativamente no $1 \underline{\mathrm{O}}$ dia de PO. No $5 \underline{\mathrm{O}}$ dia de $\mathrm{PO}$ o grupo 1 apresentou medidas significativamente maiores nos níveis xifóide $(p=0,03)$, costal $(p=0,01)$ e umbilical $(p=0,02)$ do que o grupo 2, revelando melhores resultados para o uso do Voldyne.

\section{DISCUSSÃO}

Os resultados deste estudo mostram que em todos os pacientes as cirurgias abdominais repercutiram na capacidade pulmonar, diminuindo os volumes pulmonares, o que foi evidenciado pela diminuição da expansibilidade toracoabdominal medida por cirtometria. Esses achados estão de acordo com os de Karayiannakis et al. ${ }^{19}$, para os quais a cirurgia aberta provoca uma mudança do padrão ventilatório abdominal para torácico, atribuindo a isso a queda da contribuição diafragmática ao volume corrente, devido à dor pós-operatória e disfunção pulmonar respiratória. A diminuição da expansibilidade toracoabdominal observada no 1ㅇ PO pode ser atribuída à disfunção do diafragma, dor, incisão cirúrgica e efeitos da anestesia. Para Pasquina et al.20, anestesia, disfunção do nervo frênico e o trauma cirúrgico provocam redução do funcionamento dos músculos respiratórios, levando ao decréscimo da capacidade vital forçada (CVF) e da capacidade vital por vários dias, e portanto à atelectasia.

Barisione et al. 21 relatam que na ci- rurgia abdominal alta ocorre redução nos volumes pulmonares do pré para o pós-operatório, onde a CVF, o pico de fluxo e a capacidade residual funcional são reduzidas para valores inferiores a 70\% dos obtidos no pré-operatório, os quais podem não ser restabelecidos no $5 \underline{0}$ dia de PO. A redução de todos os valores de

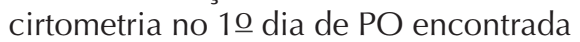
no presente estudo é consistente com esses achados, tendo-se observado perdas nas circunferências de $68,4 \%$ a 39,1\% dos valores do pré-operatório.

Gastaldi et al.22 demonstraram que exercícios respiratórios provocam a recuperação mais rápida dos volumes pulmonares e da força dos músculos respiratórios em pacientes submetidos a colecistectomia, podendo propiciar um retorno mais rápido às condições pulmonares pré-operatórias. Os resultados de Chiavegato et al.23, que estudaram as alterações da ventilação, volumes pulmonares e índice diafragmático no $\mathrm{PO}$ de colecistectomia, mostram que esses níveis retornaram aos valores basais entre o 4ํㅡ e o 6ํㅡㄹ dia de pós-operatório. No presente estudo, as circunferências médias nos níveis xifóide e umbilical no 50 PO do grupo 1 ultrapassaram os valores do pré-operatório, enquanto as médias das demais circunferências se aproximaram dos valores do pré-operatório, embora se mantivessem ainda menores.

Na comparação entre os grupos, os resultados mostraram que a recuperação da expansibilidade toracoabdominal foi maior e mais rápida no grupo 1 do que no grupo 2, sugerindo uma maior efetividade do Voldyne em relação ao padrão ventilatório em três tempos. Romanini et al. 12 compararam a espirometria de incentivo e respiração por pressão positiva intermitente (RPPI) na prevenção de complicações pulmonares pós-operatórias e observaram que a RPPI foi mais eficiente na recuperação de hipoxemia, enquanto a espirometria de incentivo foi mais eficaz na melhora da força muscular respiratória, sendo preferida pelo estímulo visual proporcionado e pela facilidade de utilização. Já Barbalho-Moulim et al. 6 sugerem que a espirometria de incentivo estimula a inspiração profunda, requisitando a atividade diafragmática. Tomich et al.24, ao comparar três tipos de exercícios respiratórios em indivíduos saudáveis, observaram mudanças no volume e tempo do padrão respiratório quando comparados aos iniciais, sendo que a utilização do Voldyne apresentou melhores resultados. Também em um estudo25 comparando o padrão respiratório e o movimento toracoabdominal durante a respiração em repouso usando três técnicas respiratórias em pacientes submetidos a gastroplastia, a espirometria de incentivo orientada a volume forneceu os melhores resultados, permitindo inspirações mais lentas e profundas.

Embora a incidência de complicações pós-cirúrgicas não tenha sido aqui estudada, os grupos não apresentaram evidencia de complicações pulmonares, sugerindo que a normalização da função pulmonar precocemente pode acarretar menor risco dessas complicações. O estudo retrospectivo de Lunardi et al. 9 evidenciou a contribuição da fisioterapia respiratória pela baixa incidência de infecções respiratórias, porém sem redução no tempo de hospitalização. No presente estudo, os pacientes tiveram alta hospitalar precoce, em torno do 50 dia PO, o que pode estar relacionado à ausência de complicações pulmonares, provavelmente decorrentes da intervenção precoce da fisioterapia pela metodologia utilizada.

Como limitação do estudo podem-se considerar a ausência de testes de função pulmonar, o reduzido número de pacientes e a ampla faixa etária, que poderiam acarretar respostas fisiológicas distintas.

\section{CONCLUSÃO}

Nos pacientes submetidos a cirurgia abdominal alta, a intervenção fisioterapêutica, pelas duas técnicas utilizadas, proporcionou recuperação gradual da dinâmica toracoabdominal. O grupo exercitado usando o dispositivo Voldyne apresentou resultados significativamente melhores do que o grupo que treinou o padrão inspiratório em três tempos, revelando a maior eficácia da espirometria de incentivo a volume. 


\section{REFERÊNCIAS}

1 Guimarães MMF, El Dib RP, Smith AF, Matos D. Incentive spirometry for prevention of postoperative pulmonary complications in upper abdominal surgery. Anesth Analg. 2009;109(5):1700.

2 Kanat F, Golcuk A, Teke T, Golcuk M. Risk factors for postoperative pulmonary complications in upper abdominal surgery. ANZ J Surg. 2007;77(3):135-41.

3 Qaseem A, Snow V, Fitterman N, Hornbake ER, Lawrence VA, Smetana GW, et al. Risk assessment for and strategies to reduce perioperative pulmonary complications for patients undergoing noncardiothoracic surgery: a guideline from the American College of Physicians. Ann Intern Med. 2006;144(8):575-80.

4 Duggan M, Kavanagh BP. Pulmonary atelectasis: a pathogenic perioperative entity. Anesthesiology. 2005;102(4):838-54.

5 Silva NLS, Piotto RF, Barboza MAI, Croti UA, Braile DM. Inalação de solução salina hipertônica como coadjuvante da fisioterapia respiratória para reversão de atelectasia no pós-operatório de cirurgia cardíaca pediátrica. Rev Bras Cir Cardiovasc. 2006;21(4):468-71

6 Barbalho-Moulim MC, Miguel GPS, Forti EMP, Costa D. Comparação entre inspirometria de incentivo e pressão positiva expiratória na função pulmonar após cirurgia bariátrica. Fisioter Pesq. 2009;16(2):166-72.

7 Dronkers J, Veldman A, Hoberg E, Waal CV, Meeteren N. Prevention of pulmonary complications after upper abdominal surgery by preoperative intensive inspiratory muscle training: a randomized controlled pilot study. Clin Rehabil. 2008;22(2):134-42.

8 Mackay MR, Ellis E, Johnston C. Randomised clinical trial of physiotherapy after open abdominal surgery in high-risk patients. Aust J Physiother. 2005;51(3):151-9.

9 Lunardi AC, Resende JM, Cerri OM, Carvalho CRF. Efeito da continuidade da fisioterapia respiratória até a alta hospitalar na incidência de complicações pulmonares após esofagectomia por câncer. Fisioter Pesq. 2008;15(1):72-7.

10 Hulzebos EH, van Meeteren NL, van den Buijs BJ, de Bie RA, Brutel de la Rivière A, Helders PJ. Feasibility of preoperative inspiratory muscle training in patients undergoing coronary artery bypass surgery with a high risk of postoperative pulmonary complications: a randomized controlled pilot study. Clin Rehabil. 2006;20(11):949-59.

11 Haeffener MP, Ferreira GM, Menna Barreto SS, Arena R, Dall'Ago P. Incentive spirometry with expiratory positive airway pressure reduces pulmonary complications, improves pulmonary function and 6-minute walk distance in patients undergoing coronary artery bypass graft surgery. Am Heart J. 2008;156(5):900.e1-8.

12 Romanini W, Muller AP, Carvalho KA, Olandoski M, Faria-Neto JR, Mendes FL, et al. The effects of intermittent positive pressure and incentive spirometry in the postoperative of myocardial revascularization. Arq Bras Cardiol. 2007;89(2):94-9.
13 Agostini P, Calvert R, Subramanian H, Naidu B. Is incentive spirometry effective following thoracic surgery? Interact Cardiovasc Thorac Surg. 2008;7(2):297-300.

14 Dias CM, Plácido TR, Ferreira MFB, Guimarães FS, Menezes SLS. Inspirometria de incentivo e breath stacking: repercussões sobre a capacidade inspiratória em indivíduos submetidos a cirurgia abdominal. Rev Bras Fisioter. 2008;12(2):94-9.

15 Parreira VF, Coelho EM, Tomich GM, Alvim AMA, Sampaio RF, Britto RR. Avaliação do volume corrente e da configuração tóraco-abdominal durante o uso de espirômetro de incentivo a volume e a fluxo, em sujeitos saudáveis: influência da posição corporal. Rev Bras Fisioter. 2004;8(1):45-51.

16 Caldeira VS, Starling CCD, Britto RR, Martins JA, Sampaio RF, Parreira VF. Precisão e acurácia da cirtometria em adultos saudáveis. J Bras Pneumol. 2007;33(5):519-26.

17 Owens WD, Felts JA, Spitznagel EL. ASA physical status classifications: a study of consistency of ratings. Anesthesiology. 1978;49(4):239-49.

18 Ide MR, Caromano FA, Dip MAVB, Guerino MR. Exercícios respiratórios na expansibilidade torácica de idosos: exercícios aquáticos e no solo. Fisioter Mov. 2007;20(2):33-40.

19 Karayiannakis A, Makri G, Mantzioka A, Karousos D, Karatzas G. Postoperative pulmonary function after laparoscopic and open cholecystectomy. Br J Anaesth. 1996;77(4):448-52.

20 Pasquina P, Tramer MR, Granier JM, Walder B. Respiratory physiotherapy to prevent pulmonary complications after abdominal surgery: a systematic review. Chest. 2006;130(6):1887-99.

21 Barisione G, Rovida S, Gazzaniga GM, Fontana L. Upper abdominal surgery: does a lung function test exist to predict early severe postoperative respiratory complications? Eur Respir J. 1997;10(6):1301-8.

22 Gastaldi AC, Magalhães, CMB, Baraúna MA, Silva EMC, Souza, HCD. Benefícios da cinesioterapia respiratória no pós-operatório de colecistectomia laparoscópica. Rev Bras Fisioter. 2008;12(2):100-6.

23 Chiavegato LD, Jardim JR, Faresin SM, Juliano Y. Alterações funcionais respiratórias na colecistectomia por via laparoscópica. J Pneumol. 2000;26(2):69-76.

24 Tomich GM, França DC, Diório ACM, Britto RR, Sampaio RF, Parreira VF. Breathing pattern, thoracoabdominal motion and muscular activity during three breathing exercises. Braz J Med Biol Res. 2007;40(10):1409-17.

25 Tomich GM, França DC, Diniz MTC, Britto RR, Sampaio RF, Parreira VF. Efeitos de exercícios respiratórios sobre o padrão respiratório e movimento toraco-abdominal após gastroplastia. J Bras Pneumol. 2010;36(2):197-204. 